\title{
Isolation of an Antigenic Oligosaccharide Fraction from Leptospira interrogans serovar canicola with a Monoclonal Antibody
}

\author{
By E. ONO, ${ }^{1}$ M. NAIKI ${ }^{2}$ AND R. YANAGAWA ${ }^{1 *}$ \\ Department of Hygiene and Microbiology ${ }^{1}$ and Department of Biochemistry ${ }^{2}$, Faculty of \\ Veterinary Medicine, Hokkaido University, Sapporo 060, Japan
}

(Received 13 September 1983; revised 24 January 1984)

\begin{abstract}
An oligosaccharide fraction containing the antigenic determinant of lipopolysaccharide antigen (TM antigen) from Leptospira interrogans serovar canicola, recognized by a monoclonal antibody (CT3) which agglutinates serovars canicola and broomi, was isolated by formic acid and successive sulphuric acid hydrolyses. Separation of the antigenic compounds was done by Bio-Gel P-2 and Sephadex G-25 gel filtration, and high-performance liquid chromatography with two different columns. The fraction finally obtained was a mixture of two oligosaccharides, both of which migrated as a single spot having a slightly higher mobility than an authentic tetrasaccharide (stachyose) on thin layer chromatography. The fraction contained rhamnose, arabinose and two major and two minor unknown sugars which were shown to be $\mathrm{N}$ - or $\mathrm{O}$-acetylated and/or $\mathrm{O}$ methylated sugars by nuclear magnetic resonance. The fraction inhibited the binding of CT3 antibody with TM antigen in enzyme-linked immunosorbent assay and microscopic agglutination of serovar canicola with the antibody. The inhibitory activity was destroyed by periodate oxidation or mild alkaline treatment, but was resistant to sodium borohydride reduction.
\end{abstract}

\section{INTRODUCTION}

There have been several reports on the isolation and characterization of leptospiral antigens (Hindle \& White, 1933; Ezell et al., 1952; Schneider, 1954; Rothstein \& Hiatt, 1956; Schricker \& Hanson, 1963; Shinagawa \& Yanagawa, 1972; Faine et al., 1974; Adler et al., 1980; Palit \& Harrison, 1980). The identification of antigenic determinant groups of leptospira forms the basis for the classification of leptospiras. They have not yet been defined, despite numerous studies (Kasai \& Yanagawa, 1974; Adachi \& Yanagawa, 1975, 1978; Tsuji et al., 1981; Kawaoka et al., 1982). The reasons for the difficulty in defining leptospiral antigenic determinants may be attributed to (1) the difficulty of isolation and purification of a serovarspecific haptenic compound, (2) the extremely small yield of such a haptenic compound and (3) the use of polyclonal antibodies for serological tests.

In previous studies of leptospiral antigen, rabbit hyperimmune sera have been used, and the serovar-specific main antigen (TM antigen) of leptospira, which has lipopolysaccharide properties, has been extracted from leptospiral organisms (Shinagawa \& Yanagawa, 1972). Ono et al. (1982a) described the production of monoclonal antibodies to TM antigen of Leptospira interrogans serovar canicola and classified them into three groups by their cross-reactivities. Group I (CT3, CT7 and CT9), group II (CT5) and group III (CT6) antibodies cross-reacted with serovars broomi, jonsis and malaya, respectively, besides the homologous cells in a microscopic agglutination test. These antigenic determinant groups on the TM antigen are completely different in their chemical properties. The group I determinant was destroyed by mild alkaline treatment with $0.1 \mathrm{M}-\mathrm{NaOH}$; it was, however, quite resistant to mild acid hydrolysis with $2 \mathrm{M}$ formic acid. On the other hand, the group II determinant was stable to the alkaline treatment, but unstable to the acid treatment (Ono et al., 1982b). The group III determinant was unstable to 
both the treatments. Therefore, monoclonal antibodies are more suitable for use in studies on antigenic determinants than polyclonal antibodies.

The present paper describes the isolation from serovar canicola of a canicola- and broomispecific oligosaccharide fraction which was defined by a monoclonal antibody (CT3). Some immunochemical properties of this fraction are also described.

\section{METHODS}

TM antigen. Leptospira interrogans serovar canicola strain Hond Utrecht IV was grown in protein-free medium (Bey \& Johnson, 1978) at $30^{\circ} \mathrm{C}$ for 1 week. Serovar-specific main (TM) antigen was extracted and purified from the organisms as previously described (Shinagawa \& Yanagawa, 1972). Briefly, lipids were removed from the wet cells with chloroform/methanol $(2: 1, \mathrm{v} / \mathrm{v})$ and then the cells were extracted with $90 \%(\mathrm{w} / \mathrm{v})$ phenol at $55^{\circ} \mathrm{C}$. The extracted lipopolysaccharides were precipitated by adding ethanol in amounts up to 50 to $80 \%(\mathrm{v} / \mathrm{v})$. The precipitated material was digested with crystalline alkaline protease $\left(750\right.$ units $\mathrm{g}^{-1}$; Nagase Biochemicals, Fukuchiyama, Japan). After dialysis, TM antigen was precipitated again by adding ethanol at $60 \%$.

Isolation of antigenic polysaccharide fraction from TM antigen. TM antigen was dissolved in $2 \mathrm{M}$-formic acid at a concentration of $10 \mathrm{mg} \mathrm{ml}^{-1}$ and heated at $100^{\circ} \mathrm{C}$ for $30 \mathrm{~min}$. The hydrolysate was filtered through a Diaflo PM10 ultrafiltration membrane (Amicon Corp., Lexington, Mass., USA). The unfiltered portion was mixed with the same volume of the acid solution and was then hydrolysed again under the same conditions. The hydrolysis and filtration procedure was repeated four times. The pooled filtrate was evaporated, applied to a Bio-Gel P-2 column $(1.2 \times 140 \mathrm{~cm})$ and eluted with pyridine/acetic acid/water $\left(1: 0.4: 100\right.$, by vol.) at a flow rate of $10 \mathrm{ml} \mathrm{h}^{-1}$. The eluate was fractionated into 50 tubes of $3 \mathrm{ml}$ each, and $100 \mu \mathrm{l}$ of each fraction was used for the quantitative determination of neutral sugars. The inhibitory activity of each fraction was determined by the inhibition test in enzyme-linked immunosorbent assay (ELISA) using $10 \mu \mathrm{g}$ of each fraction after lyophilization. The inhibitory fraction was eluted at the position of the void volume, and it was designated as the antigenic polysaccharide fraction.

Sulphuric acid hydrolysis of the antigenic polysaccharide fraction. The antigenic polysaccharide fraction was dissolved in $0.25 \mathrm{M}$-sulphuric acid at a concentration of $10 \mathrm{mg} \mathrm{ml}^{-1}$ and heated at $100^{\circ} \mathrm{C}$ for $30 \mathrm{~min}$. The hydrolysate was neutralized with barium hydroxide, and the precipitate was removed by filtration. After evaporation of the filtrate, a sample was fractionated on a Sephadex G-25 column $(1.5 \times 150 \mathrm{~cm})$ as illustrated in Fig. 1.

High-performance liquid chromatography (HPLC). The FS-2 fraction, which was obtained by gel filtration after sulphuric acid hydrolysis, was further chromatographed on columns of Shodex OH pak B-804 (Showa Denko, Tokyo, Japan), and Fine pak SIL CN (Japan Spectroscopic, Tokyo, Japan) with a Jasco TWINCLE high* pressure liquid chromatograph apparatus (Japan Spectroscopic) and a refractive index detector, Shodex SE-11 (Showa Denko). The conditions and elution profile for each chromatography are shown in Fig. 2 and Fig. 3.

Inhibition test in ELISA and microscopic agglutination inhibition test. The ELISA test with TM antigen was that developed by Ono et al. (1982a). The inhibition test in ELISA was done as follows. TM antigen was coated on each well of a 96-well microtitration plate by adding $50 \mu$ l Dulbecco's phosphate-buffered saline ( $\mathrm{pH} 7.0)$ containing $100 \mathrm{ng} \mathrm{TM}$ antigen. After non-specific binding sites of the plate had been blocked with $1 \%$ bovine serum albumin solution, a twofold serial dilution of test antigen $(25 \mu \mathrm{l}$, each fraction obtained from TM antigen by acid hydrolysis and commercially available rhamnose and arabinose) and the same volume of a 1:100 dilution of CT3 hybridoma culture medium were added to each well. The other ELISA procedures followed were those described previously (Ono et al., 1982a). The percentage inhibition was calculated as follows:

$$
\text { Inhibition }(\%)=\frac{O D_{\text {without inhibitor }}-O D_{\text {with inhibitor }}}{O D_{\text {without inhibitor }}} \times 100
$$

The microscopic agglutination inhibition test was carried out with a $1: 10$ dilution of CT3 hybridoma culture medium which agglutinated serovar canicola almost completely. The procedure was as previously described (Adachi \& Yanagawa, 1977) except inhibition of $100 \%$ agglutination was used.

Treatment of the antigenic oligosaccharide fraction. Periodate oxidation was done by dissolving the antigenic oligosaccharide fraction in $0.05 \mathrm{M}$-sodium acetate buffer $(\mathrm{pH} 5.0)$ containing $5 \mathrm{mM}$-sodium metaperiodate at a concentration of $2.5 \mathrm{mg} \mathrm{ml}^{-1}$. The mixture was kept at $4{ }^{\circ} \mathrm{C}$ for $1 \mathrm{~h}$ in a dark room and the reaction was stopped by addition of $20 \mu \mathrm{l}$ ethylene glycol $\mathrm{ml}^{-1}$.

Mild alkaline treatment was carried out by dissolving the same fraction in $0.1 \mathrm{M}-\mathrm{NaOH}$ solution at a concentration of $1 \mathrm{mg} \mathrm{ml}^{-1}$ and keeping it at room temperature for $20 \mathrm{~h}$. After neutralization with $1 \mathrm{M}-\mathrm{HCl}$, the solution was concentrated by evaporation and desalted by Sephadex G-10 column $(1 \times 75 \mathrm{~cm})$ chromatography.

Reduction with sodium borohydride was done by dissolving the fraction in $73 \mathrm{mM}-\mathrm{NaBH}_{4}$ solution at a 
concentration of $10 \mathrm{mg} \mathrm{ml}^{-1}$, and keeping it at $20^{\circ} \mathrm{C}$ for $20 \mathrm{~h}$. The remaining $\mathrm{NaBH}_{4}$ was then inactivated by the addition of a drop of glacial acetic acid, and the salt was removed by gel filtration, as described above.

Chemical analyses. Neutral sugar content was determined by the phenol/sulphuric acid reagent (Dubois et al., 1956) with glucose as the standard. Protein content was measured by the Lowry method with bovine serum albumin as the standard. Fatty acid content was determined by gas-liquid chromatography (GLC) as described previously (Naiki et al., 1975). Thin-layer chromatography (TLC) was done with silica gel 60 TLC plates (Merck). A sample $(100 \mu \mathrm{g})$ of each fraction was applied to the TLC plate and developed three times with acetone/water/methanol/chloroform $(8: 2: 1: 1$, by vol.). Sugar components were visualized by spraying with anthrone/sulphuric acid reagent and heating at $120^{\circ} \mathrm{C}$ for $15 \mathrm{~min}$. Acetylation of the antigenic oligosaccharide fraction was done with a mixture of pyridine/acetic anhydride for $12 \mathrm{~h}$ at $60^{\circ} \mathrm{C}$. After evaporation, the acetylated sample was dissolved in chloroform, applied to the TLC plate, and developed with 1-butanol/acetone/water (4:5:1, by vol.).

GLC of sugars was done as follows. A sample was hydrolysed with $1 \mathrm{M}-\mathrm{HCl}$ for $5 \mathrm{~h}$ at $100^{\circ} \mathrm{C}$, and alditol acetate derivatives of the hydrolysate were prepared by the method of Sawardecker et al. (1965). GLC was done with a glass column $(0.5 \mathrm{~cm} \times 2 \mathrm{~m})$ packed with $3 \%$ OV-225 on Supelcoport (80/100 mesh; Nishio Industry, Tokyo, Japan), at $200^{\circ} \mathrm{C}$. A GC-6A gas chromatography apparatus (Shimadzu Seisakusho, Kyoto, Japan) was used for the analysis.

Proton nuclear magnetic resonance (NMR) spectrum of the antigenic oligosaccharide fraction was recorded with a $400 \mathrm{MHz}$ JEOL FX-400 spectrometer (Nippon Denshi, Tokyo, Japan) at a concentration of $10 \mathrm{mg} \mathrm{ml}^{-1}$.

\section{RESULTS}

\section{Isolation of the antigenic oligosaccharide fraction}

Sulphuric acid hydrolysate of the antigenic polysaccharide fraction was separated into six fractions by gel filtration on Sephadex G-25 (Fig. 1). The two early fractions (FS-1 and FS-2) showed antigenic activity and inhibited the binding of CT3 antibody with TM antigen in ELISA. The FS-2 fraction, which was smaller, was further separated into two fractions by HPLC with a Shodex OH pak B 804 column (Fig. 2). The antigenic activity was recovered in the second fraction (H-2) which was then successively separated by HPLC with a Fine pak SIL CN column (Fig. 3). Of the three fractions obtained, the first (CN-1) and second (CN-2) fractions showed antigenic activity. The $\mathrm{CN}-1$ fraction was purified twice by chromatography: it then showed an anthrone-positive spot between authentic raffinose and stachyose by TLC (Fig. 4). The $\mathrm{CN}-2$ fraction migrated as a single spot having a slightly slower mobility than stachyose, but it was not recovered in sufficient amounts for further analyses. About $2 \mathrm{mg}$ of the purified $\mathrm{CN}-1$ fraction was recovered from $100 \mathrm{~g}$ of wet leptospiral cells.

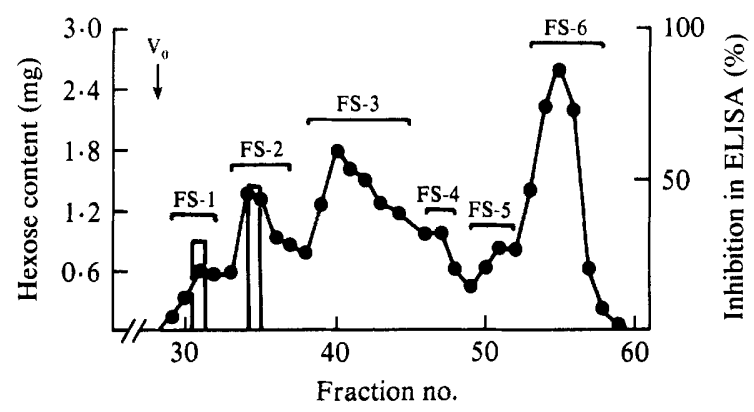

Fig. 1. Sephadex G-25 gel filtration of the sulphuric acid hydrolysate of the antigenic polysaccharide fraction. The hydrolysate $(78.5 \mathrm{mg})$ was applied to a column $(1.5 \times 140 \mathrm{~cm})$ and eluted with pyridine/acetic acid/water (1:0.4:100, by vol., pH 5.4) at a flow rate of $20 \mathrm{ml} \mathrm{h}^{-1}$. The hexose content (O) of each fraction $(3 \mathrm{ml})$ was analysed and used to divide the fraction into six subfractions (FS-1 to FS-6) by peaks of hexose content. The antigenic activity of each pooled fraction (open bars) was then determined by the inhibition test in ELISA using $10 \mu \mathrm{g}$ of each sample after lyophilization. $\mathrm{V}_{0}$, void volume. 


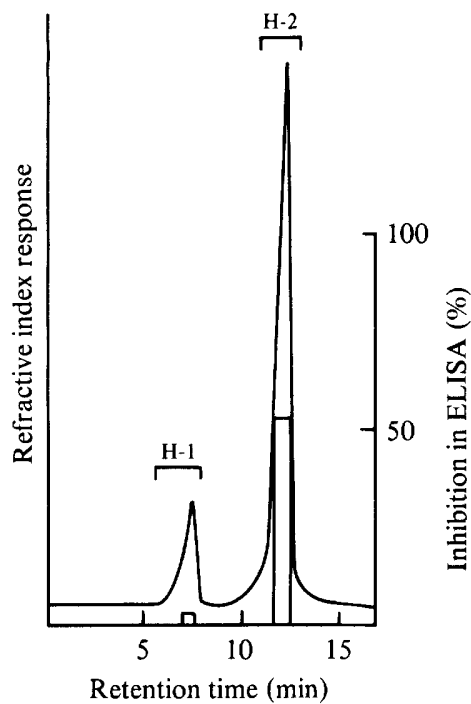

Fig. 2

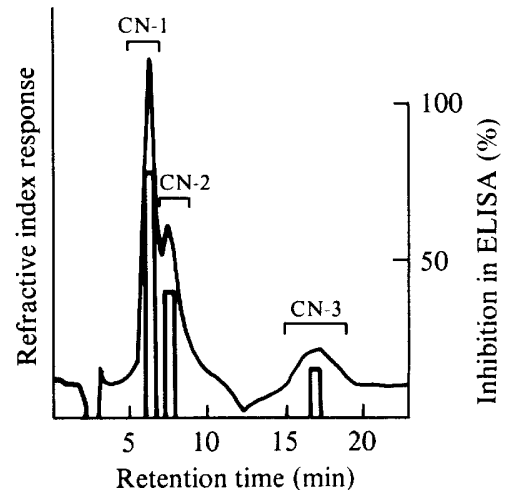

Fig. 3

Fig. 2. HPLC of the FS-2 fraction. A sample ( $2 \mathrm{mg}$ ) was applied to a Shodex OH pak B-804 column and eluted with water at a flow rate of $1.5 \mathrm{ml} \mathrm{min}^{-1}$. The antigenic activities of two major fractions $(\mathrm{H}-1$ and H-2, open bars) were determined as for Fig. 1.

Fig. 3. HPLC of the H-2 fraction. A sample $(200 \mu \mathrm{g})$ was applied to a Fine pak SIL CN column and eluted with acetone/water $(3: 1, \mathrm{v} / \mathrm{v})$ at a flow rate of $1.5 \mathrm{ml} \mathrm{min}{ }^{-1}$. The antigenic activities of three fractions (CN-1 to $\mathrm{CN}-3)$ were determined as for Fig. 1.

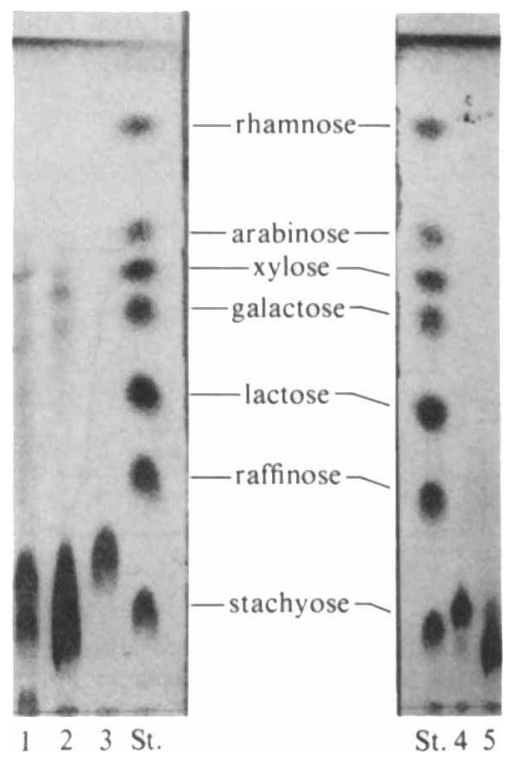

Fig. 4. TLC of each fraction. Lane 1, FS-2; lane 2, H-2; lanes 3 and 4, CN-1; lane 5, CN-2; St., authentic sugars. 


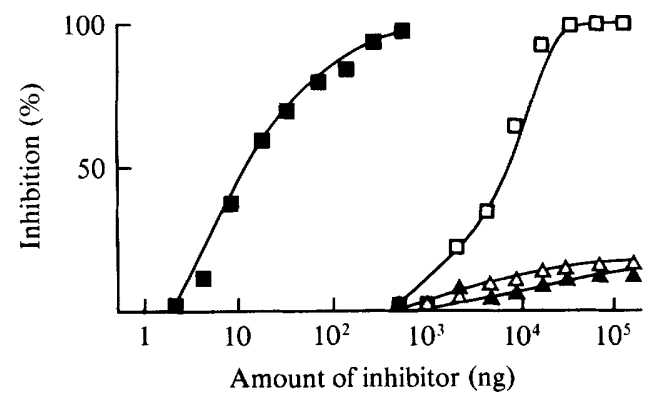

Fig. 5

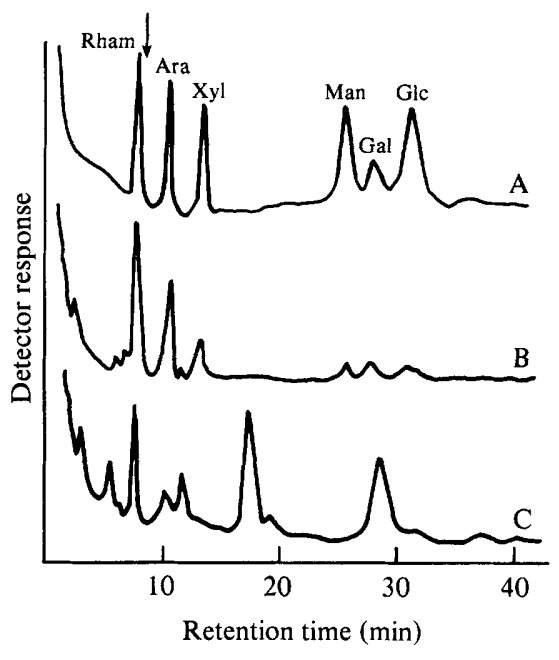

Fig. 6

Fig. 5. Antigenic activity of the $\mathrm{CN}-1$ fraction in ELISA inhibition test and the effect of periodate oxidation or mild alkaline treatment. The antigenic activity of the $\mathrm{CN}-1$ fraction $(\square)$ was compared with that of the TM antigen ( $\square)$, and the $\mathrm{CN}-1$ fraction oxidized with periodate $(\triangle)$ or treated with alkali $(\boldsymbol{A})$ and also compared in the same test.

Fig. 6. GLC of the alditol acetate derivatives prepared from authentic sugars (A), TM antigen (B) and $\mathrm{CN}-1$ fraction $(\mathrm{C})$. The arrow shows the retention time of fucose.

\section{Antigenic activity of the purified $\mathrm{CN}-1$ fraction}

By the ELISA inhibition test, the CN-1 fraction showed 500 times less inhibitory activity than intact TM antigen, but its inhibition was specific for CT3 antibody (Fig. 5). The inhibitory activity of the fraction was destroyed by periodate oxidation or mild alkaline treatment (Fig. 5), but it was stable to reduction with sodium borohydride. The $\mathrm{CN}-1$ fraction also inhibited microscopic agglutination of serovar canicola with CT3 antibody; $250 \mu \mathrm{g} \mathrm{CN}-1$ fraction gave complete inhibition, although the inhibitory potency was 100 times less than that of TM antigen.

\section{Chemical properties of the purified $C N-1$ fraction}

The molecular weight of the purified $\mathrm{CN}-1$ fraction was estimated to be $1500-1800$ by gel filtration on Bio-Gel P-2 and/or Sephadex G-15. The fraction gave a single spot by TLC as described above, but after acetylation it was separated into two spots, which overlapped each other on TLC.

The sugar composition of the fraction was analysed by GLC. The fraction contained rhamnose and two unknown sugars as major components, and arabinose and two unknown sugars as minor components (Fig. 6). From the retention time, the unknown sugars were not identical to rhamnose, fucose, arabinose, xylose, mannose, galactose or glucose. None of the unknown sugars corresponded to any major components of TM antigen. Commercially available rhamnose and arabinose did not show antigenic activity at a concentration of $150 \mathrm{mg} \mathrm{ml}^{-1}$, indicating that the antigenic determinant may be associated with the unknown sugars.

The proton NMR spectrum of the fraction indicated that it contained four $\mathrm{N}$ - and/or $\mathrm{O}$-acetyl groups (1.94, 1.97, 1.99 and 2.04 p.p.m.) and four $O$-methyl groups (2.89, 3.46, 3.52 and 3.59 p.p.m.).

\section{DISCUSSION}

The nature of the antigenic determinant group contained in TM antigen has not been characterized in detail. Adachi \& Yanagawa $(1975,1978)$ reported that the determinant groups 
of TM antigen of serovars kremastos $\mathrm{Kyoto}$ and hebdomadis are proteinaceous because they were destroyed by protease digestion. Tsuji et al. (1981) and Kawaoka et al. (1982) succeeded in isolating the serovar-specific polysaccharide fraction from serovars canicola and kremastos Kyoto, without any protein or fatty acid. They concluded that the antigenic determinants were associated with carbohydrate. They used polyclonal rabbit hyperimmune sera for the studies; consequently they could not isolate such a low molecular weight antigen as $\mathrm{CN}-1$ oligosaccharide fraction. Our previous studies (Ono et al., 1982a) on monoclonal antibodies to Leptospira interrogans indicated that TM antigen of serovar kremastos Kyoto has more than 10 antigenic determinants, each of which is common to other serovars, and that TM antigen of serovar canicola has more than three different determinants. Therefore, we utilized a monoclonal antibody for the isolation of a single determinant. CT3 antibody (group I) was used, because it recognized the acid-stable antigenic determinant. Formic acid hydrolysis of TM antigen produced an antigenic fraction with a molecular weight of about 3000 . In order to isolate smaller fragments, further hydrolysis was done with sulphuric acid.

The CN-1 oligosaccharide fraction was finally obtained by several chromatographic separations. Unfortunately, the fraction was still a mixture of two oligosaccharides which could be separated by TLC only when they were acetylated. Alkaline treatment for deacetylation, however, easily destroyed the antigenic activity of the fraction. In the present study, we attempted to analyse the mixture. The two oligosaccharides seemed to be tetra- or pentasaccharides, from their mobilities in TLC. One or both contained rhamnose, arabinose and two major unknown sugars which could not be detected in the intact TM antigen. Since no inhibition was detected by rhamnose and arabinose, the antigenic determinant may be associated with the unknown sugars.

The antigenic determinant to CT3 antibody is considered to be a very small constituent in TM antigen. In the present study, the final yield of the purified $\mathrm{CN}-1$ fraction was only $0.2 \%$ of the TM antigen preparation. Only $6 \mathrm{mg} \mathrm{CN}-1$ fraction was obtained from $3 \mathrm{~g} \mathrm{TM}$ antigen, which was extracted from $300 \mathrm{~g}$ wet cells collected from approximately 1000 litres of leptospiral culture. Such a small yield of the antigenic oligosaccharide fraction makes it difficult to elucidate the serovar-specific chemical structure. When a sufficient amount of the fraction is available and good methods for separation of the two oligosaccharides have been developed, the exact chemical structure of the antigenic determinant will be elucidated.

The authors thank Dr S. Gasa, Cancer Research Institute, Hokkaido University, for recording the proton NMR spectrum and for his enthusiastic discussion about it, and Prof. S. Kubo, Faculty of Veterinary Medicine, Hokkaido University, for his invaluable support and discussion. This work was supported by Grants-in-Aid for Scientific Research no. 548062 , no. 56360026 and no. 58440012 from the Ministry of Education, Science and Culture of Japan.

\section{REFERENCES}

ADaChI, Y. \& Yanagawa, R. (1975). Possible role of protein(s) as antigenic determinant of the typespecific main antigen of Leptospira kremastos strain Kyoto. Infection and Immunity 11, 1325-1331.

ADACHI, Y. \& YANAGAWA, R. (1977). Inhibition of leptospiral agglutination by the type-specific main antigen of leptospiras. Infection and Immunity 17, 466-467.

Adachi, Y. \& YanaGawa, R. (1978). Nature of antigenic determinant of serovar-specific antigen of Leptospira interrogans serovar hebdomadis. Microbiology and Immunology 22, 523-533.

Adler, B., Faine, S. \& Yanagawa, R. (1980). Comparative studies on two antigens (F4 and TM) extracted from Leptospires. Journal of Clinical Microbiology 12, 7-9.
Bey, R. F. \& Johnson, R. C. (1978). Protein-free and low-protein media for the cultivation of Leptospira. Infection and Immunity 19, 562-569.

Dubois, M., Gilles, K. A., Hamilton, J. K., Rebers, P. A. \& SMITH, F. (1956). Colorimetric method for determination of sugars and related substances. Analytical Chemistry 28, 350-356.

Ezell, S. B., HoAG, W. G., WARNer, A. R., YAGeR, R. H. \& GoCHENOUR, W.S. (1952). Soluble specific leptospiral complement-fixing antigens. Proceedings of the Society for Experimental Biology and Medicine 80, 220-223.

Faine, S., Adler, B. \& Palit, A. (1974). Chemical, serological and biological properties of a serotypespecific polysaccharide antigen in Leptospira. Australian Journal of Experimental Biology and Medical Science 52, 311-319. 
Hindle, E. \& White, P. B. (1933). A soluble specific substance in spirochaetes. Proceedings of the Royal Society B114, 523-529.

Kasai, N. \& YanaGaWA, R. (1974). Studies on the antigenic determinant group of the type-specific antigen of Leptospira canicola. Zentralblatt für Bakteriologie, Parasitenkunde, Infektionskrankheiten und Hygiene (Abteilung I, Originale A) 228, 533-541.

KaWaOKa, Y., NaIKI, M. \& Yanagawa, R. (1982). Isolation of antigen-active components from leptospiral serovar-specific lipopolysaccharide antigen by mild acid hydrolysis. Japanese Journal of Veterinary Science 44, 473-478.

Naiki, M., Fong, J., Ledeen, R. \& Marcus, D. M. (1975). The structure of the human erythrocyte blood group $\mathrm{P}_{1}$ glycosphingolipid. Biochemistry 14, 48314836.

Ono, E., NaIki, M. \& Yanagawa, R. (1982a). Production and characterization of monoclonal antibodies to lipopolysaccharide antigen of Leptospira interrogans serovar kremastos and canicola. Zentralblatt für Bakteriologie, Parasitenkunde, Infektionskrankheiten und Hygiene (Abteilung I, Originale A) 252, 414-424.

Ono, E., Tsuji, M., Naiki, M. \& Yanagawa, R. $(1982 b)$. Production of monoclonal antibodies to lipopolysaccharide antigen of Leptospira interrogans serovar kremastos and canicola, and studies on the antigenic determinant groups with monoclonal antibodies. Japanese Journal of Bacteriology 37, 126 (in Japanese).
Palit, A. \& Harrison, P. M. (1980). Serological and chemical interrelationship of antigens from Leptospira interrogans serovar canicola. Journal of General Microbiology 117, 141-146.

Rothstein, N. \& HiatT, C. W. (1956). Studies of the immunochemistry of leptospires. Journal of Immunology 77, 257-265.

SaWARdecker, J. S., Slonecker, J. H. \& Jeanes, A. (1965). Quantitative determination of monosaccharides and their alditol acetates by gas liquid chromatography. Analytical Chemistry 37, 16021604.

SCHNEIDER, M. D. (1954). Isolation and chemical composition of complement-fixing antigens from leptospires. Proceedings of the Society for Experimental Biology and Medicine 85, 32-37.

SCHRICKER, R. L. \& Hanson, L. E. (1963). Precipitating antigens of leptospires. I. Chemical properties and serological activity of soluble fractions of Leptospira pomona. American Journal of Veterinary Research 24, 854-860.

Shinagawa, M. \& Yanagawa, R. (1972). Isolation and characterization of a leptospiral type-specific antigen. Infection and Immunity 5, 12-19.

Tsuj, M., Kawaoka, Y., NaIKI, M. \& Yanagawa, R. (1981). Isolation of antigenically active components from leptospiral serovar-specific lipopolysaccharide antigen by alkaline treatment. Microbiology and Immunology 25, 949-957. 